\title{
MOBILE LEARNING: A UTILIZAÇÃO DO APLICATIVO NEARPOD EM UMA DISCIPLINA DE ZOOLOGIA III NO CURSO DE LICENCIATURA EM CIÊNCIAS BIOLÓGICAS
}

\section{MOBILE LEARNING: THE USE OF THE APP NEARPOD IN A DISCIPLINE OF ZOOLOGY III IN THE LICENSEE COURSE IN BIOLOGICAL SCIENCES}

\author{
Gabriel Henrique de Lima ${ }^{1}$; Ricardo Sérgio da Silva ${ }^{2}$; Kênio Erithon Cavalcante Lima ${ }^{3}$; \\ Patricia Smith Cavalcante ${ }^{4}$
}

\section{RESUMO}

O presente artigo tem como intuito analisar se o Nearpod se configura como um instrumento tecnológico capaz de proporcionar aulas atrativas e dinâmicas para os estudantes da disciplina de Zoologia III, no curso de Licenciatura em Ciências Biológicas da Universidade Federal de Pernambuco. A escolha de tal recurso se deu devido as suas características particulares que possibilitam ao docente trabalhar em sala de aula na perspectiva da Mobile Learning, uma vez que pode oportunizar a utilização de smartphones em sala de aula. Nossa hipótese é que a utilização de dispositivos móveis nas estratégias didáticas dos professores pode gerar maiores reflexões por parte dos licenciandos, no que diz respeito à aprendizagem móvel, visto que, tal temática é extremamente importante no âmbito da formação docente e muitas vezes não tem espaços de discussões nas licenciaturas. Como percurso metodológico, este trabalho apresenta um caráter quanti-qualitativo e configura-se como uma pesquisa do tipo participante. Dentre os sujeitos envolvidos no estudo, temos uma amostragem de 32 estudantes que participaram da pesquisa e um professor que leciona na disciplina de Zoologia III. Os dados foram coletados durante um encontro de quatro horas no período noturno com os estudantes da referida disciplina. Durante a intervenção realizamos a aplicação de dois questionários disponibilizados via Google Docs, direcionados para os referidos sujeitos. Os resultados indicaram que o Nearpod contribuiu significativamente para a promoção de uma aula dinâmica e engajadora, uma vez que os estudantes se mostraram bastante envolvidos durante todo o processo e também devido ao fato de o professor utilizar as diversas ferramentas didáticas presente na plataforma para tornar a aula mais atrativa aos estudantes. Dentre as dificuldades encontradas durante a intervenção, podemos chamar atenção para os problemas de infraestrutura tecnológica, que gerou alguns empecilhos durante a intervenção, mas que logo foram contornados. Nessa perspectiva, esse estudo abre espaço para oportunizar maiores discussões acerca do Mobile Learning em sala de aula, que se configura como um tipo de aprendizagem que ocorre através das tecnologias móveis, em qualquer temo e espaço.

Palavras-Chave: Aprendizagem Móvel, Nearpod, Smartphones.

\begin{abstract}
The aim of this article is to analyze whether Nearpod is a technological instrument capable of providing attractive and dynamic classes for the students of the Zoology III course in the Degree in Biological Sciences of the Federal University of Pernambuco. The choice of this feature was due to its particular characteristics that allow the teacher to work in the classroom in the

\footnotetext{
${ }^{1}$ Mestrando em Educação Matemática e Tecnológica - EDUMATEC, Universidade Federal de Pernambuco, gabrieldelima900@gmail.com

${ }^{2}$ Doutorando em Ciências Biológicas UFPE/CB, Universidade Federal de Pernambuco, profricardosergio.bio@gmail.com

${ }^{3}$ Doutor em Educação, Universidade Federal de Pernambuco, keclima@ig.com.br

${ }^{4}$ Doutora em Educação, Universidade Federal de Pernambuco, patricia3smith@ gmail.com
} 
perspective of M-Learning, once this app is configured as a tool that can enable the use of smartphones in the classroom. Thus, it is believed that the use of mobile devices in the didactic strategies of the teachers can generate more reflections on the part of the students with respect to the mobile learning, since, this subject is extremely important in the educational scope and often does not have spaces of discussions in the degrees. The methodology of this work is quantitative-qualitative character and it is configured as a participative research type. Among the subjects involved in the study, we have a sample of 32 students who participated in the research and a teacher who teaches in the discipline of Zoology III. The data were collected during a four hour meeting in the evening with the students of this discipline. During the intervention we carried out the application of two questionnaires made available through Google Docs directed to the said subjects. The results indicated that Nearpod contributed significantly to the promotion of a dynamic and engaging classroom, since students were very involved throughout the process and also because the teacher used the various didactic tools present in the platform to make the more attractive class to students. Among the difficulties encountered during the intervention, we can draw attention to the problems of technological infrastructure that generated some difficulties during the intervention, but that soon was bypassed. In this perspective, this study opens the door to further discussion about M-Learning that is configured as a type of learning that occurs through mobile technologies.

Keywords: Mobile Learning, Nearpod, Smartphones.

\section{INTRODUÇÃO}

Vivemos em um contexto social onde as Tecnologias da Informação e Comunicação (TIC) se encontram presentes em nosso cotidiano adquirindo um caráter ubíquo e central em nossas vidas. Ao permitir trocas de informações de maneira instantânea com qualquer pessoa do mundo, as tecnologias modificam assim, a forma como nos comunicamos e interagimos em sociedade. Além disso, devido as constantes evoluções que as tecnologias vêm sofrendo dentro de nossa sociedade, torna-se extremamente necessário que os sujeitos estejam abertos as inovações e consigam se adaptar as diversas reconfigurações sociais causadas pelos avanços tecnológicos.

Pouco tempo atrás, para que pudéssemos trocar e-mails ou fazer uma chamada de vídeo, por exemplo, tínhamos com ter acesso a um computador. Embora tal ferramenta fosse prática e permitisse buscar informações sobre qualquer tipo de assunto, ainda tinha um empecilho que era o fato desse equipamento ser caro e nem um pouco portátil.

Com o surgimento e popularização dos dispositivos móveis a exemplo dos smartphones, agora se tornava possível ter acesso às informações em qualquer lugar a qualquer hora assumindo assim um caráter de onipresença proporcionado pelas tecnologias móveis. Desse modo, tendo em vista que dispositivos móveis já fazem parte da realidade de muitas pessoas devido a fatores como seu barateamento, surge então um conceito muito atrelado ao meio educacional chamado de aprendizagem móvel, na qual, a partir de tais dispositivos é possível transformar a forma como se aprende. 
Em vista disso, muitas ferramentas educacionais foram criadas com diversas finalidades e dentre elas o aplicativo Nearpod, que segundo Valletta (2014), pode ser definido como um aplicativo multiplataforma centrado na aprendizagem móvel, uma vez que, com ele, é possível que os estudantes possam acessar a aula em tempo real com um código gerado sempre que uma aula estiver sendo transmitida pelo professor. Por apresentar inúmeras ferramentas que permitem ao docente a criação de diversas configurações de aulas, esse aplicativo possibilita o desenvolvimento de aulas dinâmicas e engajadoras para seus alunos.

Conhecendo as diversas possibilidades utilização do Nearpod no contexto educacional, e nos apoiando em trabalhos como o de Moura (2015) que trazem resultados positivos acerta do aplicativo, destacamos aqui a questão de pesquisa norteadora desse estudo: É possível desenvolver aulas atrativas e engajadora a partir do aplicativo Nearpod em uma disciplina de Zoologia III no curso de Licenciatura em Ciências Biológicas? No intuito de alcançar tal entendimento do objeto estudado, temos como objetivo geral analisar se o aplicativo Nearpod configura-se como uma ferramenta tecnológica capaz de proporcionar aulas atrativas e dinâmicas para os estudantes da referida disciplina.

\section{FUNDAMENTAÇÃO TEÓRICA}

De acordo com a Unesco (2014), cada dia mais as tecnologias móveis vem se tornando uma realidade na vida dos cidadãos, pois, graças a fatores como barateamento dos smartphones torna-se possível para sujeitos que vivem em condições econômicas menos favorecidas terem acesso a tais dispositivos. Segundo dados da Fundação Getúlio Vargas, em abril deste ano o Brasil já contava com um quantitativo de 220 milhões smartphones ativos em todo país, chegando a uma média de cerca de um dispositivo por habitante, passando assim o quantitativo de computadores que somava cerca de 174 milhões (contabilizando computadores de mesa, tablets e notebooks).

A partir desses estudos, podemos ver o grande potencial pedagógico por trás dessas tecnologias móveis, uma vez que, são ferramentas que permitem ao usuário personalizar sua aprendizagem de acordo com seu perfil e interesse, além de permitir que o sujeito possa aprender em qualquer lugar sem a necessidade de está necessariamente em uma sala de aula.

Mesmo sabendo do grande potencial que as tecnologias móveis apresentam para o campo educacional, vemos que ainda existem inúmeros desafios que permeiam a sua inserção de maneira efetiva. Dentre os diversos fatores, podemos citar, por exemplo, o despreparo docente para lhe dar com essas tecnologias, como também carência de infraestrutura tecnológica que dê suporte a atividades como essas. De Acordo com Ferreira (2015), um dos 
grandes desafios no momento é conseguir unir o aprendizado ao contexto das tecnologias móveis, pois, além dos fatores citados acima, ainda existe muita resistência para a inserção dessa cultura da mobilidade no campo educacional. Um exemplo bastante preocupante é a Lei $\mathrm{n}^{\mathrm{o}}$ 15.507, de 21 de maio de 2015 que regulamenta o uso de aparelhos celulares nos espaços escolares. Segundo essa lei:

Art. $1^{\mathrm{o}}$ Fica proibido o uso de aparelhos celulares e equipamentos eletrônicos nos estabelecimentos de ensino públicos ou privados, no âmbito do Estado de Pernambuco, nos seguintes termos:

I - nas salas de aula, exceto com prévia autorização para aplicações pedagógicas;

II - nos demais espaços, exceto se no "modo silencioso" ou para auxílio pedagógico.

Em vista disso, faz-se pertinente refletirmos a cerca da seguinte questão: será que os cursos de formação de professores estão formando profissionais aptos a atuarem nesse novo contexto que se encontra a educação brasileira? Esta é a nova realidade educacional, uma realidade onde os alunos não dependem exclusivamente do professor para ter acesso à informação. Essa informação se encontra na palma de suas mãos e que por isso, cabe ao professor, compreender essa nova realidade educacional e está preparado para ela.

Em vista disso, o aplicativo Nearpod, enquanto ferramenta didática facilitadora da aprendizagem permite ao docente ampliar seu leque de possibilidades oportunizando a utilização dos smartphones em sala de aula de forma consciente e produtiva, uma vez que, se bem utilizado, esse recurso pode, segundo Moura (2015), envolver os estudantes na aula permitindo assim, uma aprendizagem atrativa e engajadora, além de possibilitar que o professor possa monitorar em tempo real o desempenho dos estudantes.

Dentre as diversas ferramentas que estão ao alcance do professor para montar sua aula podemos destacar, por exemplo, modelos tridimensionais dos mais variados assuntos, desde estruturas celulares 3D até construções históricas como o "Stonehenge". Além disso, ainda é possível criar simulações sobre diversos temas para que os estudantes acompanhem em seus smartphones. Outra possibilidade está no fato do docente ter a liberdade de anexar sites na apresentação, PDFs, vídeos do YouTube e até fazer transmissões ao vivo através do Twitter.

No que diz respeito ao processo avaliativo, ele pode ocorrer ao longo de toda aula, uma vez que, todas as atividades que forem realizadas pelos alunos serão salvas, possibilitando que o professor acompanhe posteriormente o desempenho de cada estudante. Dentre as diversas possibilidades avaliativas presentes na plataforma podemos destacar ferramentas como Quizzes, questões discursivas e colaborativas, votações, teste de memória, questões para preencher espaços em branco e perguntas que permitem aos alunos responderem desenhando suas respostas em seus smartphones. 


\section{METODOLOGIA}

O presente estudo apresenta uma abordagem quanti-qualitativa e caracteriza-se como uma pesquisa participante que segundo Gil (1999), evidencia-se pela interação entre o pesquisador e o pesquisado, permitindo assim, uma maior compreensão dos fenômenos analisados à medida que nesse tipo de abordagem, o pesquisador torna-se parte integrante do estudo, possibilitando que o mesmo analise de maneira mais rica os elementos subjetivos encontrados no campo da pesquisa.

Dentre os sujeitos envolvidos no estudo, temos uma amostragem de 32 estudantes que participaram da pesquisa e um professor que leciona na disciplina de Zoologia III, ofertada pelo curso de Licenciatura em Ciências Biológicas da Universidade Federal de Pernambuco do Centro Acadêmico de Vitória UFPE - CAV no período de 2017.1. Tal disciplina é de caráter obrigatório e é ofertada no quarto período do curso no horário noturno.

A disciplina de Zoologia III não foi escolhida por acaso, trata-se de uma disciplina que apresenta números de reprovações significativos e que contém uma grande carga de conteúdo atrelado a sua matriz curricular, por isso, faz-se importante intervenções como essa para mostrarmos ao professor que é possível diversificar suas estratégias metodológicas de maneira simples e de forma que permitam aos estudantes aprenderam com uma abordagem mais prazerosa.

Com relação ao recurso utilizado na pesquisa, destacamos que para a realização da intervenção foi necessário que tanto o professor quanto os alunos se familiarizassem com o aplicativo Nearpod. Para isso, solicitamos que o docente criasse uma conta na página do Nearpod (www.nearpod.com), pois, só assim seria possível preparar as aulas que seriam posteriormente disponibilizadas nos smartphones dos estudantes através de seu computador. Para os estudantes, foi necessário que eles baixassem o aplicativo disponível tanto para Android quando para IOS, ambos com tamanho de 26,02 MB e $94 \mathrm{MB}$ respectivamente.

A primeira etapa da pesquisa constituiu-se em definir com o professor da disciplina qual conteúdo seria escolhido para ser trabalhado com o auxílio do Nearpod e por questão de praticidade, o docente da disciplina de Zoologia III resolveu escolher o assunto sobre Myriapoda uma vez que, seria o próximo conteúdo a ser trabalhado com os estudantes. Dessa forma, após o professor da disciplina ceder os materiais que iriam ser utilizados em suas aulas, tivemos a tarefa de organiza-los e adaptá-los aos moldes da plataforma Nearpod, adicionando diversas ferramentas ao corpo da apresentação disponibilizadas pelo professor, tais como quizzes, vídeos, enquetes, modelos tridimensionais, etc. Ao fim da montagem da aula na 
plataforma do Nearpod reenviamos para que o professor pudesse pensar suas aulas baseado no que foi desenvolvido na plataforma.

Após a conclusão da primeira etapa, seguimos para execução da aula, onde foi necessária uma sala que contasse com conexão a internet satisfatória, uma vez que só é possível utilizar o aplicativo se os usuários estiverem conectados em alguma rede de internet. Além disso, para que a aula pudesse acontecer era preciso que os estudantes tivessem em mãos seus dispositivos móveis com o aplicativo devidamente baixado para que assim pudessem ter acesso à aula. No planejamento da aula foi reservado um tempo de 5 a 10 minutos para que os alunos que não conseguissem baixar o app por algum motivo tivessem o suporte do pesquisador para resolver eventuais problemas que surgissem, tais como incompatibilidade, memória insuficiente nos dispositivos, etc.

A terceira etapa caracterizou-se pela aplicação de dois questionários, um direcionado para o professor e outro para os estudantes, que por sua vez foram disponibilizados através da ferramenta de formulários da Google. Tais questionários apresentavam questões de múltipla escolha, questões semiestruturadas e também escala de opiniões (Escala Likert), onde a partir disso, foi possível fazer diversas análises, tanto de cunho quantitativo, utilizando as questões de múltipla escolha e Escala Likert, quanto qualitativo a partir das questões discursivas, nas quais foram investigadas a partir da Análise de Conteúdo que nos permite fragmentar as diversas ideias postas pelo sujeito, organiza-las sistematicamente e assim ter um entendimento único sob um dado fenômeno (BARDIN, 2011).

\section{RESULTADOS E DISCUSSÃO}

Os resultados apresentados nesta pesquisa são frutos de uma intervenção realizada em uma turma de 32 alunos da disciplina de Zoologia III em um curso de Licenciatura em Ciências Biológicas. Os dados aqui coletados foram positivos e apontaram para o Nearpod como um aplicativo capaz de proporcionar aulas dinâmicas e interessantes para os estudantes, à medida que, a partir das diversas ferramentas presentes neste aplicativo torna-se possível que o professor tenha um leque de possibilidades a sua disposição.

\section{Relação Nearpod e Aprendizagem}

A partir da presente pesquisa, constatamos que o aplicativo Nearpod contribuiu significativamente para um melhor aproveitamento dos estudantes na aula de Myriapoda da disciplina de Zoologia III. Tal afirmação é possível de ser verificada a partir do gráfico 1 que 
abordava a seguinte indagação: "Você considera que o recurso didático (app Nearpod) contribuiu para o entendimento do conteúdo trabalhado? Em uma escala de 1 a 10, 1 sendo ruim, 5 regular e 10 excelente".

Gráfico1: Questionário dos estudantes que buscou compreender se o Nearpod contribui para o entendimento do conteúdo trabalhado. Fonte: Própria

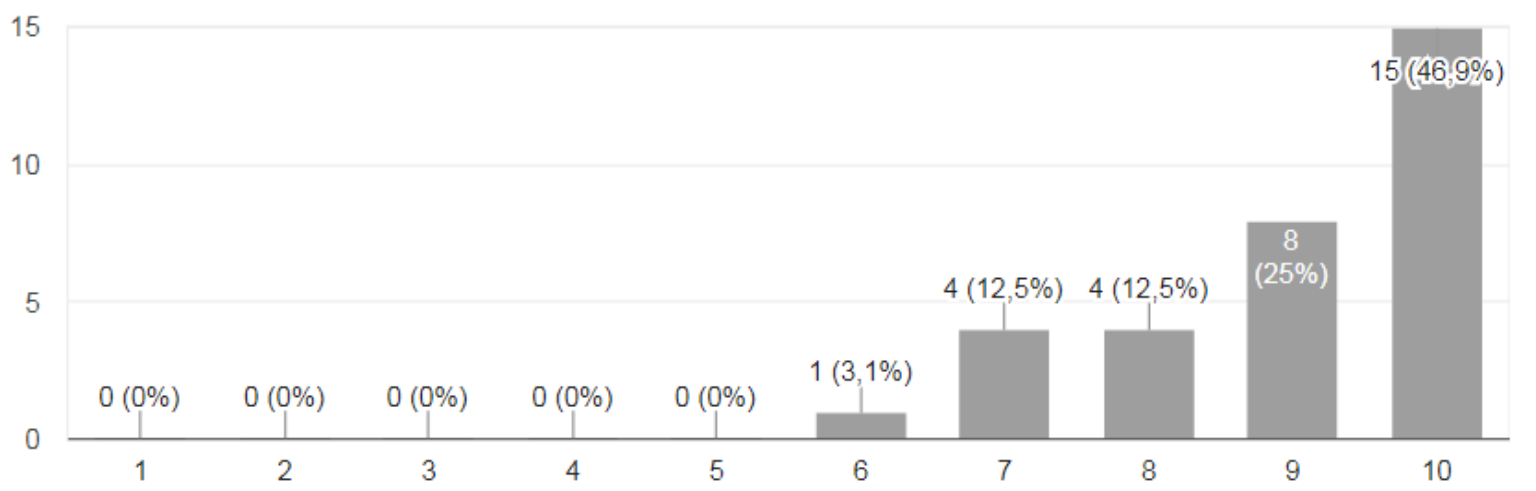

Um dos principais comentários que os estudantes fizeram referente ao Nearpod foi devido a sua capacidade de tornar a aula mais dinâmica. No decorrer da aula os alunos chamaram atenção para a importância de o professor retomar uma dada ideia que acabou de trabalhar na forma de perguntas ou até de vídeos contextualizados com o tema. Permitindo assim, que os alunos reforçassem o conteúdo que acabaram de estudar. Segundo Morán (1995), ao utilizar recursos como vídeos em sala de aula, torna-se possível criar um ambiente de entretenimento que permite um maior engajamento dos alunos com os assuntos estudados, mas para que isso seja possível, o autor destaca que esse recurso deve ser utilizado em sincronia com o que está sendo trabalhado e não como "tapa-buraco" ou de maneira descontextualizada do assunto.

Um fato bastante interessante no aplicativo estava no fato do professor poder lançar perguntas no decorrer da aula e monitorar as respostas dos alunos em tempo real. Isso permitiu que o docente soubesse quais alunos estavam com dificuldade em avançar no conteúdo e assim pudesse buscar outros meios para abordar o assunto.

Tais perguntas eram expressas pelos estudantes de diversas formas, tanto em textos, como também por meio de desenhos. Tais respostas eram desenvolvidas em conjunto de maneira colaborativa, corroborando assim os achados de Delacruz (2014), na qual a autora mostra que a partir das atividades desenvolvidas com o auxílio do Nearpod, foi possível incentivar o trabalho colaborativo dos estudantes e também o espirito competitivo, pois como as respostas dos alunos seriam mostradas na projeção para serem discutidas em conjunto, os estudantes buscaram resolver tais atividades na melhor forma possível. 


\section{Dificuldade com Infraestrutura}

Um dos principais problemas encontrados durante a realização da atividade foi relacionado à infraestrutura tecnológica do campus. Embora a universidade dispusesse de uma conexão com a internet disponível para todos os estudantes, tal rede não conseguia abranger toda área do local, existiam várias salas que não contavam com o sinal de internet e a sala de aula do quarto período de Ciências Biológicas era uma delas.

Por esse motivo, não tivemos condições de executar o que havíamos planejado, uma vez que, só fomos saber deste empecilho no momento em que chegamos à sala. Por isso, foi necessário adiar a intervenção para a semana seguinte, atrapalhando todo calendário acadêmico da disciplina. Assim, para conseguir realizar a atividade, solicitamos junto ao núcleo de tecnologia da universidade um roteador para utilizarmos em um dos laboratórios de ensino da instituição, já que nesses laboratórios existiam pontos de internet que poderiam ser ligados a esses equipamentos para ser distribuídos aos estudantes.

Na semana seguinte, antes de iniciarmos a aula, ocorreram outros transtornos pontuais, pois alguns alunos se esqueceram de instalar o aplicativo em seus dispositivos e, além disso, o roteador que estávamos utilizando não suportava tantos equipamentos conectados de uma só vez, por isso, foi solicitado aos estudantes que não conseguiram ter acesso a internet que se juntassem com os colegas que tinham o acesso tanto por meio do sinal Wi-Fi, quanto por seus pacotes de dados.

Devido a tais inconvenientes, só foi possível iniciar a aula depois que esses contratempos foram resolvidos, fazendo com que o professor perdesse vinte e cinco minutos até tudo se normalizar. No entanto, vale salientar que o planejamento feito pelo professor e pelo pesquisador foi executado com êxito, uma vez que contávamos com as quatro aulas da noite para poder realizar a intervenção.

Com base nesses problemas vivenciados durante nossa intervenção, constatamos que inúmeros são os desafios que o docente tem que enfrentar para conseguir inserir as TIC em suas práticas pedagógicas. Inúmeros são os autores que evidenciam a necessidade de uma formação tecnológicas desses docentes, no entanto, só isso não é o suficiente. Faz-se necessário que as instituições de ensino ofereçam condições estruturais básicas para que o trabalho do professor possa ser realizado com êxito (PINHEIRO 2003). Em contrapartida, notamos que o compromisso dos professores não está apenas em se qualificarem frente às tecnologias presentes na sociedade, mas sim em desenvolver competências e habilidades para lhe dar com 
as diversas variáveis que recursos como esses podem trazer. Ao se preparar para as diversas condições que lhe esperam em sala de aula, esses profissionais acabam adquirindo um perfil de criativo e autônomo (COSTA, 2017).

\section{Nearpod e Portabilidade}

Um ponto que merece destaque diz respeito à portabilidade dos estudantes. De todos os sujeitos envolvidos na pesquisa, apenas um não apresentava telefone celular compatível com o aplicativo Nearpod, corroborando assim as pesquisas feitas pela Fundação Getúlio Vargas de São Paulo (FGV-SP) e Unesco (2014), onde nos mostra que os dispositivos móveis estão presentes na vida de grande parte dos estudantes brasileiro e que por isso podem ser considerados como grandes aliados no processo educativo, devido as inúmeras possibilidades pedagógicas que eles podem trazer para o processo de ensino e aprendizagem.

\section{Importância do Uso de TIC}

Buscando compreender como os estudantes veem o uso de tecnologias em sala de aula, disponibilizamos no questionário aplicado uma questão discursiva a fim de entender se os sujeitos enxergam às tecnologias como algo essencial para o processo educativo. Dos respondentes, mais de $90 \%$ dos estudantes concordaram com tal questionamento, porém com algumas ressalvas como é possível observar na fala o aluno 1: "Não diria essencial, mas poderia ser um bom recurso para o aprendizado dos alunos, pois seria pra eles "uma coisa nova " na sala de aula, seria interessante para mostrar o conteúdo de uma forma mais lúdica e prática."

A partir dessa fala, é possível identificar que o estudante tem consciência de que a tecnologia pode ser uma grande aliada do professor permitindo que o mesmo diversifique suas estratégias didáticas a fim de tornar as aulas mais interessantes e prazerosas para o alunado, no entanto, vale salientar segundo as ideias de Vilas Boas e Barbosa (2013) que devemos ter cuidado para que a tecnologia no âmbito pedagógico não se torne apenas um recurso que prenderá a atenção dos alunos e será divertida para os mesmos. É preciso ter um propósito para sua inserção em sala de aula.

Em contra partida, não podemos ver a tecnologia como algo revolucionário que irá garantir a aprendizagem do aluno, pois a tecnologia por si só não garante isso, o que dará possibilidade para que o aluno aprenda de maneira mais significativa é como o professor utilizará as inúmeras possibilidades que estão ao seu alcance, incluindo as possibilidades 
tecnológicas, para assim chegar ao fim pretendido (MAGALHÃES, 2018).

Essas reflexões merecem muita atenção e maiores discussões, sobretudo, nos cursos de formação de professores, como é o caso do curso de Licenciatura em Ciências Biológicas investigado nesta pesquisa, pois, na questão que buscava compreender se os estudantes viam as tecnologias como algo essencial para o processo de ensino, muitas foram as falas dos estudantes que iam de encontro as ideias colocadas pelo aluno 2: "Com certeza que a tecnologia é essencial na educação, pois com ela a interação e participação ativa do aluno será alcançada de maneira mais expressiva”. Nessa fala, vemos que o estudante enxerga a tecnologia não apenas como uma ferramenta, mas sim como um instrumento que por si só irá garantir a aprendizagem, porém como colocado por Magalães (2018) o simples fato de inserção das tecnologias sem fins pedagógicos bem definidos não favorecem em nada a construção do conhecimento.

\section{Perspectiva Docente}

No que diz respeito às considerações feitas pelo professor no questionário aplicado, vimos que o docente considerou o Nearpod como uma ótima ferramenta para se trabalhar em sala de aula e, ademais, o professor ainda alegou que a partir desta pesquisa ele pode perceber que é possível utilizarmos os dispositivos móveis em sala de aula de maneira proveitosa.

Além disso, quando indagado se conhecia o aplicativo e se pretendia continuar utilizando em suas aulas o professor nos deixou as seguintes considerações: "Não conhecia, gostaria de conhecer mais e aplicar. Porém é um recurso que depende de muitas variáveis. A sua utilização devido à indisponibilidade de uma rede rápida, dos celulares dos alunos fez com que o meu planejamento curricular acabasse atrasando".

\section{CONCLUSÕES}

A partir dos resultados colhidos em nossa pesquisa, vimos que é possível utilizar os dispositivos móveis, sobretudo o aplicativo Nearpod, de maneira consistente e contextualiza no âmbito institucional, uma vez que tal recurso se mostrou eficiente na promoção de aulas dinâmicas e atrativas para os estudantes.

Inúmeros foram os desafios que permearam nosso estudo, no entanto, tais adversidades serviram para nos mostrar que se faz necessário investir cada vez mais em infraestrutura tecnológica nas instituições de ensino, pois, para que possamos ter professores criativos e dispostos a utilizar as diversas possibilidades que as tecnologias podem oferecer é importante que esses sujeitos tenham as condições básicas para colocarem suas ideias em prática. 
Com relação aos estudantes envolvidos na pesquisa, tais sujeitos se mostraram bastante engajados e satisfeitos com a intervenção. A partir do questionário aplicado e de nossa análise holística, foi possível identificar que o Nearpod contribuiu para o entendimento do conteúdo trabalhado, uma vez que, tal constatação veio tanto das respostas dos alunos ao questionário quanto da nossa observação do comportamento dos estudantes durante a intervenção. Desse modo, concluímos que os objetivos propostos nesta presente pesquisa foram alcançados.

\section{REFERÊNCIAS}

BARDIN, L. Análise de conteúdo. São Paulo: edições 70, 2011.

COSTA, C. 'Ninguém sabe ainda como formar professores inovadores', diz educadora que trabalhou na Apple e com Obama. BBC Brasil, São Paulo, 11 jan. 2017. Disponível em: $<$ https://goo.gl/BWYr7h>. Acesso em 13 de agosto de 2018.

DELACRUZ, S. Using Nearpod in elementary guided reading groups. TechTrends, 2014. p.62-69.

FERREIRA, D. F. M. A. Aprendizagem Móvel no Ensino Superior: o uso do Smartphone por alunos do Curso de Pedagogia. 2015. 107 f. Dissertação (Mestrado em em Educação Matemática e Tecnológica) - Centro de Educação, Universidade Federal de Pernambuco, Pernambuco, Recife, 2015.

GIL, A. C. Métodos e técnicas de pesquisa social. 5. ed. São Paulo: Atlas, 1999.

LIMA, B. Brasil já tem mais de um smartphone ativo por habitante, diz estudo da FGV. O Estado de São Paulo, São Paulo, 19 abr. 2018. Disponível em: 〈https://goo.gl/dgvfvW〉. Acesso em 13 de agosto de 2018.

MAGALHÃES, V. B. Tecnologia e Educação: o uso das tecnologias de mídia no ambiente escolar. São Carlos: Congresso Internacional de Educação e Tecnologias / Encontro de Pesquisadores em Educação A Distância, v. 4, n. 1, 2018.

MORÁN, J. M. O vídeo na sala de aula. Comunicação e Educação. São Paulo, 1995.

MOURA. A. Nearpod: uma solução integrada para avaliação, apresentação e colaboração. In: CARVAlHO, A. A. A. Apps para dispositivos móveis: Manual para professores formadores e bibliotecários. Lisboa: República Portuguesa, 2015. p.239-245.

PERNAMBUCO, Lei no 15.507, de 21 de maio de 2015. Disponível em: <https://goo.gl/bHRoQU>. Acesso em: 13 de agosto de 2018.

PINHEIRO, L. V. R. P. Comunidades científicas e infra-estrutura tecnológica no Brasil para uso de recursos eletrônicos de comunicação e informação na pesquisa. Revista Ciência da informação, Brasília, v. 32, n. 3, p. 62-73, 2003.

UNESCO. Organização das Nações Unidas para a Educação, a Ciência e a Cultura. 
Diretrizes de políticas para a aprendizagem móvel. Paris: UNESCO, 2014. Disponível em: <https://goo.gl/9sS6Py> Acesso em: 12 ago. 2018.

VALLETTA, D. Gui@ de aplicativos para a educação básica: uma investigação associada ao uso de tablets. Fortaleza: EdUECE, 2014.

VILAS BOAS, J.; BARBOSA, J. C. O uso de manipuláveis na participação dos alunos em uma aula de matemática. Em Teia, Recife v. 4, n. 3, p. 01-17, 2013. 